AIAA-2002-1464

\title{
Probabilistic Design of a Plate-Like Wing to Meet Flutter and Strength Requirements
}

\author{
W. Jefferson Stroud*, T. Krishnamurthy*, and Brian H. Mason ${ }^{\dagger}$, \\ NASA Langley Research Center \\ Hampton, VA 23681-2199 \\ Steven A. Smith ${ }^{\dagger}$ \\ Vehicle Technology Directorate - Army Research Laboratory \\ NASA Langley Research Center \\ Hampton, VA 23681-2199 \\ and \\ Ahmad S. Nasert \\ Lockheed Martin Space Operations \\ NASA Langley Research Center \\ Hampton, VA 23681-2199
}

\begin{abstract}
$\underline{\text { Abstract }}$
An approach is presented for carrying out reliability-based design of a metallic, plate-like wing to meet strength and flutter requirements that are given in terms of risk/reliability. The design problem is to determine the thickness distribution such that wing weight is a minimum and the probability of failure is less than a specified value. Failure is assumed to occur if either the flutter speed is less than a specified allowable or the stress caused by a pressure loading is greater than a specified allowable. Four uncertain quantities are considered: wing thickness, calculated flutter speed, allowable stress, and magnitude of a uniform pressure load.
\end{abstract}

The reliability-based design optimization approach described herein starts with a design obtained using conventional deterministic design optimization with margins on the allowables. Reliability is calculated using Monte Carlo simulation with response surfaces

\footnotetext{
*Aerospace Engineer, Analytical and Computational Methods Branch, Senior Member, AIAA

${ }^{\dagger}$ Aerospace Engineer, Analytical and Computational Methods Branch, Member AIAA

${ }^{+}$Aerospace Engineer, Aerodynamics, Structures, and Materials Department

Copyright $(2001$ by the American Institute of Aeronautics and Astronautics, Inc. No copyright is asserted in the United States under Title 17, U.S. Code. The U.S. Government has a royalty-free license to exercise all rights under the copyright claimed herein for Governmental Purposes. All other rights are reserved by the copyright owner.
}

that provide values of stresses and flutter speed. During the reliability-based design optimization, the response surfaces and move limits are coordinated to ensure accuracy of the response surfaces.

Studies carried out in the paper show the relationship between reliability and weight and indicate that, for the design problem considered, increases in reliability can be obtained with modest increases in weight.

\section{$\underline{\text { Introduction }}$}

Advanced aerospace vehicles of the future will be built to meet design requirements that are defined in terms of the likelihood, or probability, that the vehicle will perform as intended. In this paper, that likelihood is referred to as the reliability, and the associated design approach is referred to as reliability-based design.

Reliability-based design has several advantages over traditional deterministic design. For example, with these methods, measures of reliability and safety are available during the design process and for the final design. This information allows the designer to produce a consistent level of safety and efficiency throughout the vehicle-no unnecessary over-designs in some areas. As a result, designers may be able to save weight while maintaining adequate reliability and safety. In addition, in reliability-based design it is possible to determine the sensitivity of the reliability to design changes that can be linked to changes in cost. Designers could carry out trade studies involving 
reliability, weight, and cost. Also, for the same cost, it may be possible for aerospace vehicles to be made more reliable and safer than with traditional design approaches, or, for the same safety and reliability, the vehicle may be made at a lower cost. The primary disadvantages of reliability-based design are that a substantial amount of both data and computations are needed.

To address these disadvantages, this paper provides an example of data collection and illustrates computational techniques that help make reliability-based design tractable. In addition, this paper provides insight into reliability-based design for an example design problem involving two disciplines-aeroelasticity and stress analysis.

This paper presents a design procedure by which a plate-like wing with variable thickness can be designed to be minimum weight and to meet both a subsonic flutter requirement and a separate strength requirement. However, unlike earlier, deterministic work (e.g., Refs. 1-4), this design procedure considers several uncertainties and accounts for them by using a probabilistic approach. Also, instead of an inequality constraint on failure (flutter speed too low and/or stress too high), the inequality constraint is on the probability of failure. The design variables define the wing thickness distribution.

First, the paper provides a description of the design problem. Then the details of the approaches used and the results of the studies are presented. The studies show the relationship between reliability and weight and indicate that, for the design problem considered, increases in reliability can be obtained with modest increases in weight.

\section{Description of Wing Design Problem}

The objective of the study is to obtain the minimum-weight plate-like wing that satisfies design requirements on both flutter and strength. This example was chosen because a similar metal plate can serve as the center portion, and primary stiffness, of a wind tunnel model. The appropriate airfoil shape can be created by bonding balsa wood or a foam material to the metal plate and tooling the combination. The thickness distribution of the metal plate can be tailored so that the complete model has the desired stiffness distribution.

The wing planform is shown in Figure 1. The wing semispan is $60 \mathrm{in}$., the wing root is $24 \mathrm{in}$., the tip is 12 in., and the sweep of the leading edge is 14 degrees.
The wing thickness distribution is taken to be quadratic in both the chordwise and spanwise directions. The quadratic thickness distribution is defined by the thicknesses at the nine locations shown in Figure 2. Locations 4, 5, and 6 are at mid semispan. Locations 2, 5, and 8 ane at the mid points of the local chord. These nine thicknesses are the design variables. The expression for the thickness distribution is presented and explained in Appendix A. The minimum gage requirement for the thickness is 0.150 in.

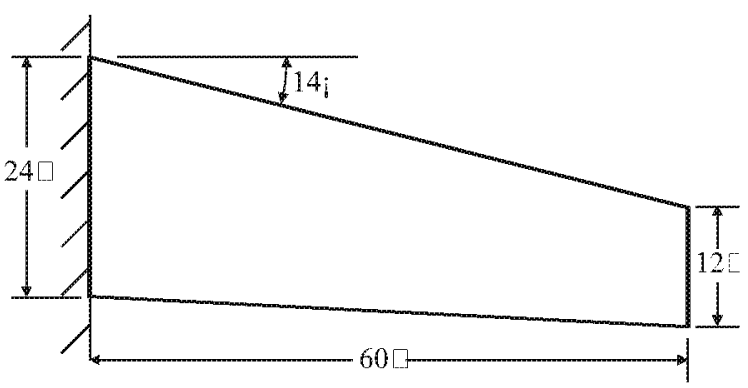

Figure 1. Planform dimensions of metal plate-like wing.

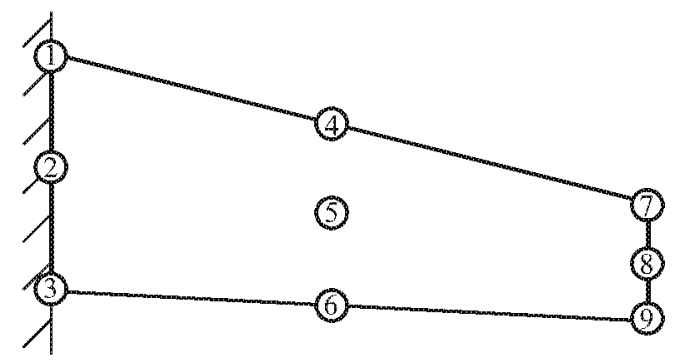

Figure 2. Location of nine thicknesses that define the thickness distribution of the plate-like wing.

There is one flutter speed requirement and one strength requirement. The flutter speed requirement is subsonic. The strength requirement is applied to each finite element. The loading for the strength analysis is uniform pressure over the surface of the wing. The wing planform is fixed; design variables control the thickness distribution. There are four uncertainties: wing thickness, calculated flutter speed, allowable stress, and magnitude of the uniform pressure load. Instead of an inequality constraint on failure (flutter speed too low and/or stress too high), the inequality constraint is on the probability of failure.

The computer code MSC/NASTRAN was used to calculate both the stresses (Ref. 5) and the flutter speed (Ref. 6). The finite element grid was obtained by 
dividing the wing into 18 equally-spaced segments spanwise and 9 segments chordwise - making a total of 162 finite elements. The finite element grid is shown in Figure 3. The finite element that was used in the stress analysis is denoted CQUAD8.

For the flutter analysis, the KE method, which is a modified $\mathrm{K}$ method, was used. Eight flutter modes were calculated. The frequency and damping for each mode were calculated at 99 reduced frequencies. The flutter speed for each of these modes was obtained from the reduced frequency at which the damping becomes negative. The Doublet-Lattice method was used to calculate the required aemodynamic matrices. The aerodynamic grid was obtained by dividing the wing into 10 equally-spaced segments spanwise and 5 segments chordwise. The aerodynamic grid is shown in Figure 4.

Values of non-random quantities that were used in the wing design study are given in Table 1 . These quantities were used in the stress analysis and in the flutter analysis. Definitions of the four types of random quantities that were used in carrying out the probabilistic wing design study are given in Figure 5 . The nine random thicknesses $\mathrm{t}_{\mathrm{i}}$ (random) were obtained by taking the input values of $t_{i}$ and adding a random increment from the uniform distribution shown in Figure 5. Note that the mean value of the increment is not zero. Further discussion of randomness in $t_{i}$ is given in Appendix B.

The fourth type of random quantity defined in Figure 5 is the calculated flutter speed, which depends on the thickness distribution (defined by $\mathrm{t}_{\mathrm{i}}$ (random)), on the aerodynamic grid, and on the flutter analysis method (e.g., KE and PK). To account for the uncertainties associated with the aerodynamic grid and analysis method, an increment from a uniform distribution was added to the flutter speed calculated using $t_{i}$ (random). The increment was based on the assumption that the flutter speed calculated with other aerodynamic grids and by other methods is within $\pm 3 \%$ of the flutter speed calculated with the grid and method used here.

Table 1. Values of the non-random quantities that define the wing design problem

\begin{tabular}{|c|c|}
\hline $\begin{array}{l}\text { Quantities associated with wing metallic material } \\
\text { Modulus of elasticity } \\
\text { Density } \\
\text { Poisson's ratio }\end{array}$ & $\begin{array}{l}10 \times 10^{6} \mathrm{psi} \\
0.10 \mathrm{lb} / \mathrm{in}^{3} \\
0.30\end{array}$ \\
\hline $\begin{array}{l}\text { Quantities associated with flutter analysis } \\
\text { Reference air density } \\
\text { Mach Number } \\
\text { Reference Chord } \\
\text { Structural damping (g) } \\
\text { (over the range of frequencies from } 0-1000 \mathrm{~Hz} \text { ) }\end{array}$ & $\begin{array}{l}\text { 0.0023 slug/ } \mathrm{ft}^{3} \\
0.45 \\
24 \text { in. } \\
0.01\end{array}$ \\
\hline
\end{tabular}

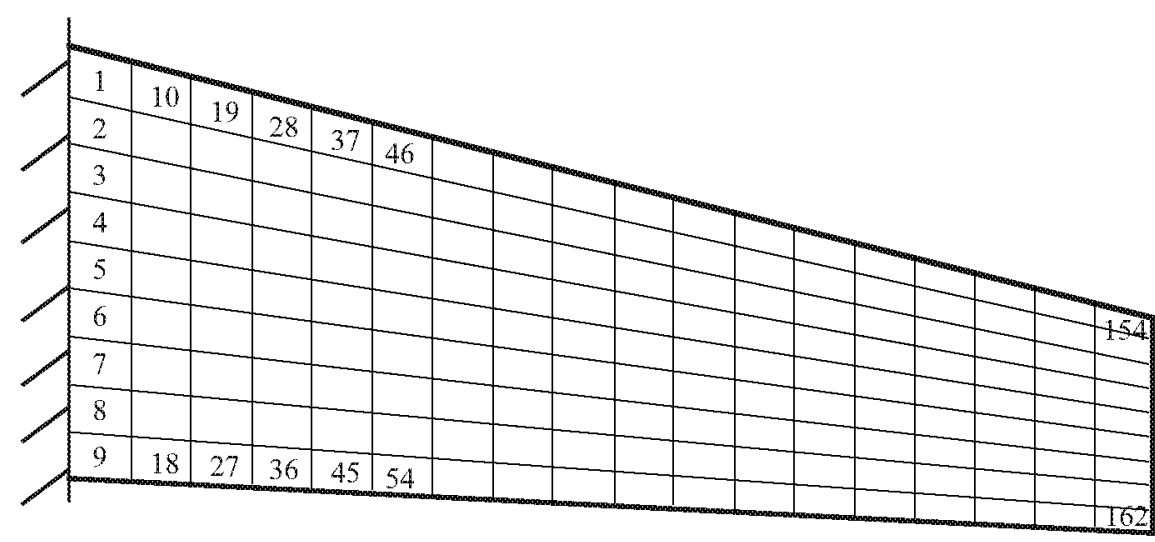

Figure 3. Finite element grid with pattem of element numbers. 


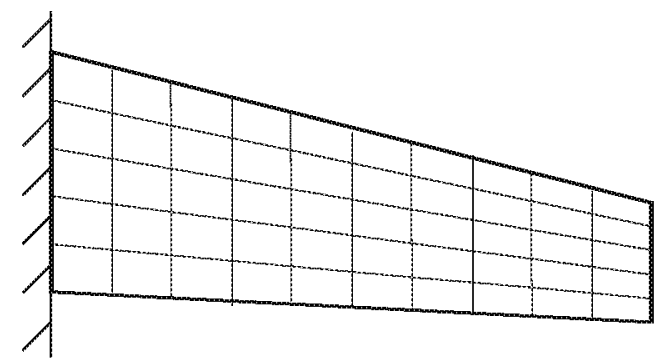

Figure 4. Aerodynamic grid.

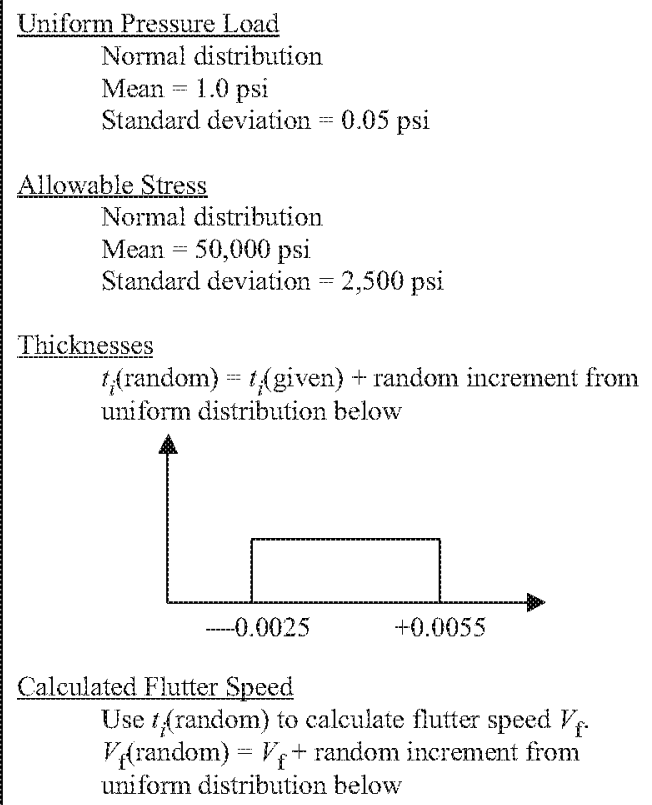

$-0.0025$

$+0.005$

Calculated Flutter Speed

Use $t_{i}$ (random) to calculate flutter speed $V_{\mathrm{f}}$ $V_{\mathrm{f}}($ random $)=V_{\mathrm{f}}+$ random increment from uniform distribution below

Figure 5. Definitions of the four random quantities used in the probabilistic design problem.

With the above information, the wing design problem can be stated as follows. Find the values of the thicknesses $t_{i}(i=1, \ldots, 9)$ such that wing weight is a minimum and the probability of system failure $\left(\mathrm{P}_{\mathrm{f}}\right)$ is less than a specified value (e.g., 0.01). The objective function is the wing weight calculated using $t_{i}$ rather than the mean value of the wing weight calculated using $t_{i}$ (random). System failure is assumed to occur if any constraint is violated. The constraints are (1) the flutter speed must be greater than a specified allowable and (2) the von Mises stress at the centroid of any element must be less than the specified allowable. The design problem is an example of a series system in which there are many failure modes, any of which can cause the system to fail.

\section{Design Approach}

The design approach was a combination of an analyzer (MSC/NASTRAN), an optimizer (DOT, Ref. 7), and FORTRAN and $C$ code for approximate analysis and probabilistic analysis. First, the method for selecting the starting point for the reliability-based optimization is discussed. Next, the analyses used to perform the reliability-based design are described. Then, possible sources of error and methods for enror alleviation in this approach are discussed.

The first step was to carry out a deterministic optimization with margins on the allowables. The allowables for both the deterministic and probabilistic optimization are shown in Table 2. Standard methods were used to carry out the deterministic optimization.

Table 2. Allowables for both deterministic and probabilistic optimization

\begin{tabular}{|c|c|c|}
\hline \multirow{2}{*}{ Allowable } & \multicolumn{2}{|c|}{ Type of optimum } \\
\cline { 2 - 3 } & Deterministic & Probabilistic \\
\hline Stress & $44,000 \mathrm{psi}$ & $\begin{array}{c}50,000 \\
\text { (Mean })\end{array}$ \\
\hline Flutter Speed & $550 \mathrm{ft} / \mathrm{sec}$ & $540 \mathrm{ft} / \mathrm{sec}$ \\
\hline
\end{tabular}

The deterministic optimum served as a starting point for the probabilistic optimization. Monte-Carto simulation (MCS) was used to calculate the reliability. Approximate analysis techniques in the form of response surfaces were used to provide estimates of the flutter speed and the stresses. The stresses were the von Mises stresses evaluated at the centroid of each of 162 finite elements. A response surface was generated for this stress in each element. Therefore, with the response surface for the flutter speed, a total of 163 response surfaces were used during the calculation of the reliability. (The type of response surface used is discussed in Appendix C.) In calculating the reliability, MCS received all input from the response surfaces rather than from MSC/NASTRAN.

Two sources of error, MCS and the response surface calculations, affected the calculation of the 


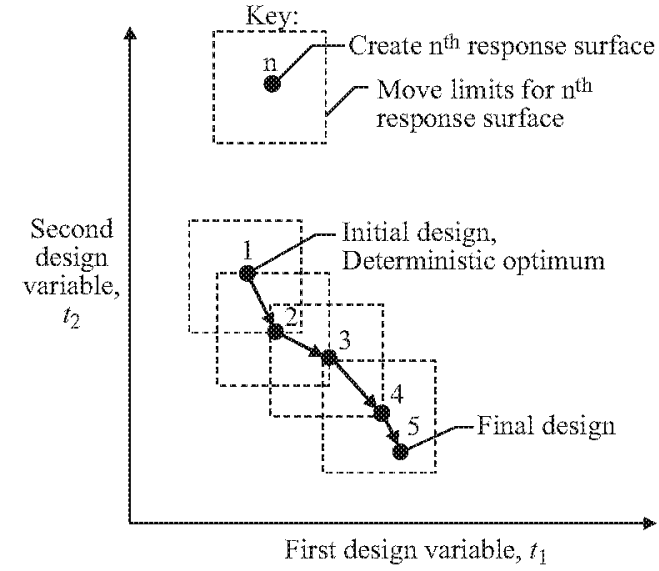

Figure 6. Move limit strategy during probabilistic optimization.

reliability. In the following two paragraphs, these two sources of emor are discussed along with the approach taken to minimize their effect

In MCS, the reliability is computed from an integer count of the number of simulations that meet the reliability requirements. A large number of simulations are more likely to accurately reflect the distribution of the underying random variables. As a result, a large number of simulations are more likely to provide an accurate prediction of the reliability. In addition, the optimizer requires derivatives of the reliability with respect to the design variables. These derivatives are computed by central differences using small changes in the design variables. Small changes in the design variables may not change the count in an integer-based reliability calculation unless a large number of simulations are performed. In this paper, to maintain the required accuracy, one million MCS trials were used for each estimate of the reliability.

In generating a response surface, points are selected to provide an accurate estimate of the response in the region of interest. Initially, several sets of response surfaces with different sampling region sizes were generated to study the accuracy of the response surfaces. Emors in the response surfaces were too large to use the entire design space for a sampling region. Because the error in the response surfaces must be

\footnotetext{
${ }^{\S}$ It is possible to estimate the emor associated with a Monte Carto simulation. For example, if a Monte Carto simulation with one million trials indicates a reliability of $\mathbf{0 . 9 9 0 0}$, there is a $95 \%$ probability that the true answer is between 0.9898 and 0.9902 (nefs. 8,9).
}

much less than the variation in the responses due to uncertainty, it was necessary to use response surface sampling zones no larger than 0.01 inches for this problem.

For this design problem, multiple response surfaces are used. When the optimizer selects a new design, the move limits are adjusted to match the region over which the response surfaces are accurate. The move limit strategy is illustrated in Figure 6, which shows a hypothetical optimization search in 2-D design space. Once the move limits are reached, new response surfaces are generated, a new move dinection is calculated, and new move limits ane set.

As mentioned previously, minimum gage thickness was chosen to be 0.150 in. Before calling $\mathrm{MSC} /$ NASTRAN to generate the response surfaces, the wing thickness was calculated at the comers of each finite element. If the thickness was found to be less than minimum gage, it was set equal to 0.150 in.

\section{$\underline{\text { Results and Discussion }}$}

First, the deterministic optimization is discussed. Then, the probabilistic optimization is discussed. Results from the deterministic optimization served as a starting point for the probabilistic optimization.

\section{Deterministic optimization}

In this study, two different deterministic optimum designs were found with approximately the same weight. For each of these two designs, the weight, flutter speed, largest von Mises stress (and element number), and the design variables are presented in Table 3 .

Contour plots showing the thickness distributions for the two designs are presented in Figure 7. Substantial parts of both wings are at minimum gage $(0.150$ in). Both designs are thick along the leading edge near midsemispan. In addition, design 1 is thick at the root near mid-chord, while design 2 is thick at the root near the trailing edge. Plots showing the von Mises stress at the centroid of each element are presented in Figure 8. The stress distributions are similar, in many respects, to the thickness distributions. In design optimization such as this, the procedure adjusts thicknesses to simultaneously improve both the local strength and the load paths. The load paths for designs 1 and 2 are different.

To explore why there appear to be two converged deterministic optimum designs, a technique was used 
Table 3. Quantities associated with deteministic optima

\begin{tabular}{|c|c|c|}
\hline Quantity & Optimum 1 & Optimum 2 \\
\hline Weight (lb) & 30.820 & 30.818 \\
\hline Flutter speed $(\mathrm{ft} / \mathrm{sec})$ & 550.06 & 549.61 \\
\hline $\begin{array}{l}\text { Largest von Mises stress (psi) } \\
\text { at centroid of element number: }\end{array}$ & $\begin{array}{l}43,999 . \\
5\end{array}$ & $\begin{array}{l}44,012 . \\
46\end{array}$ \\
\hline $\begin{array}{l}\text { Design variables (in) } \\
t_{1}\end{array}$ & 0.15000 & 0.15000 \\
\hline $\mathrm{t}_{2}$ & 0.54880 & 0.43030 \\
\hline$t_{3}$ & 0.15000 & 0.51194 \\
\hline$t_{4}$ & 0.60145 & 0.56690 \\
\hline$t_{5}$ & 0.19433 & 0.21366 \\
\hline$t_{6}$ & 0.15000 & 0.15000 \\
\hline$t_{7}$ & 0.15000 & 0.15000 \\
\hline$t_{8}$ & 0.15000 & 0.15000 \\
\hline t9 & 0.15000 & 0.15000 \\
\hline
\end{tabular}
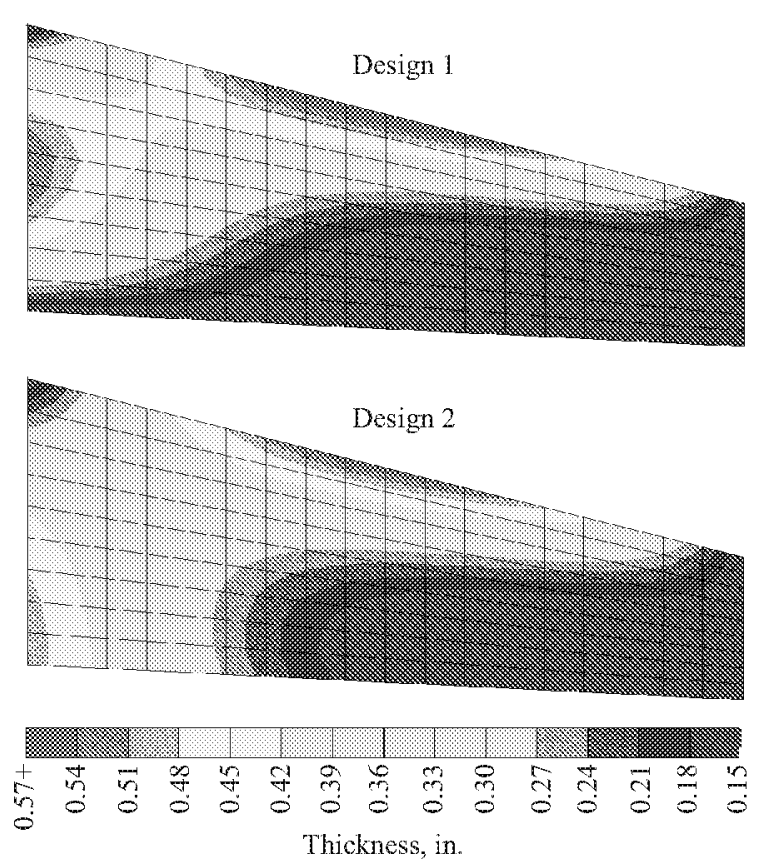

Figure 7. Thickness distribution for optimum deterministic designs 1 and 2.

that is similar to a technique used in Reference 10. A plane containing three designs - the two deterministic optimum designs plus a third design - is passed through design space. For the approach used herein, the third design has design variables that are two percent larger
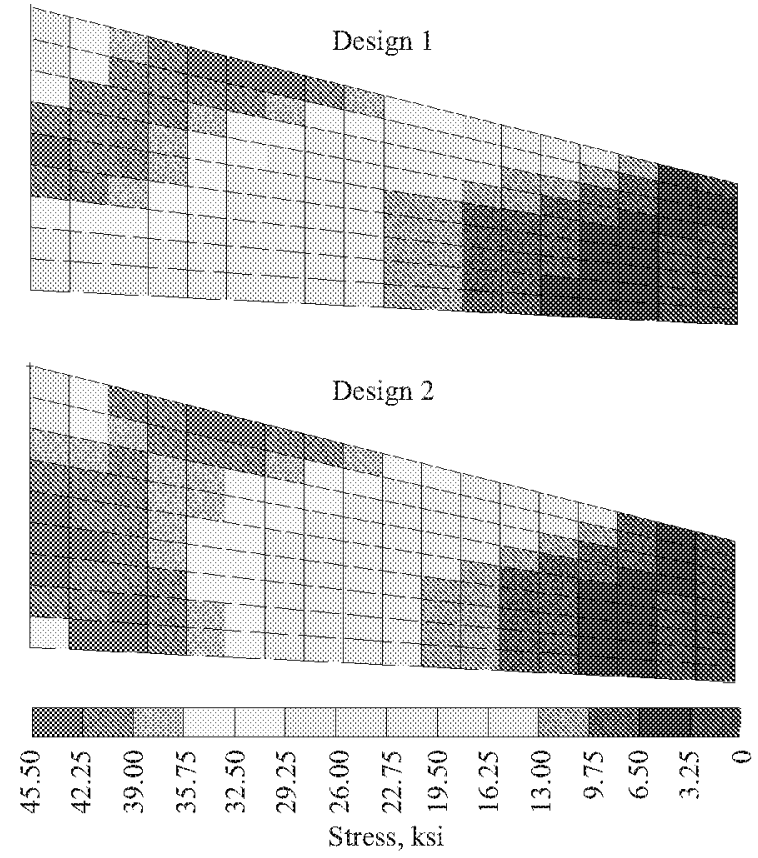

Figure 8. Von Mises stress at centroid of each finite element for optimum deterministic designs 1 and 2.

than the average of the comesponding design variables of the two deterministic optimum designs. On this plane, weight contours and constraint boundaries can be superimposed. Such a plot provides insight into various interactions in design space. 


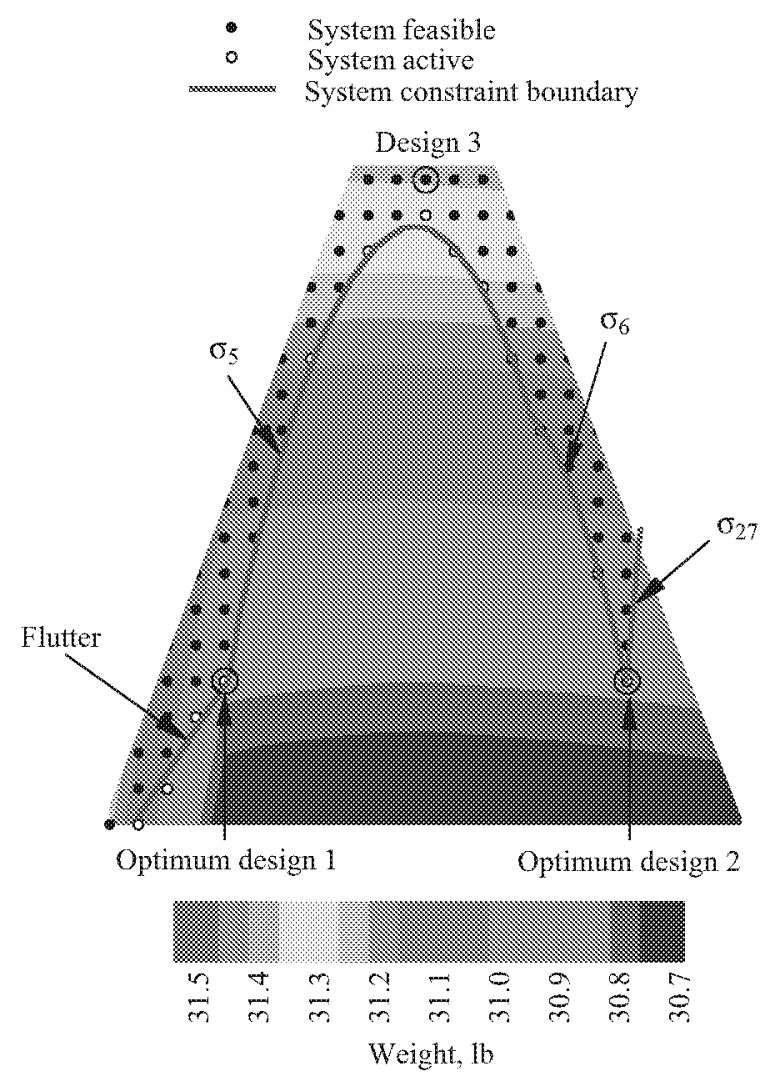

(a) Constraints are shown as a system constraint boundary that incorporates all constraints.
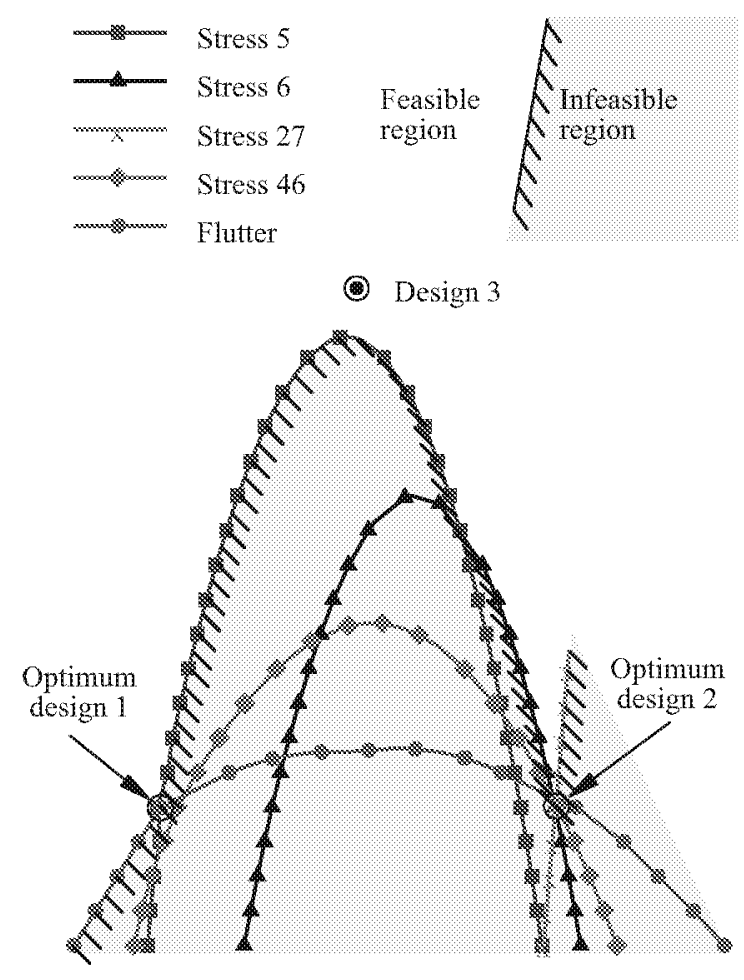

(b) Several individual constraints ane shown. Weight contours are not shown.

Figure 9. Plane through design space with optimum deterministic designs 1 and 2, weight contours, and constraints.

Such a chart is presented in Figure 9. In Figure 9a the weight is indicated by the color. Optimum deterministic design 1 is at the lower left, optimum deterministic design 2 is at the lower right, and the third design is at the top of the trapezoid. The curve denoted "System constraint boundary" is the envelope of the flutter constraint and the stress constraint in all elements. The combination of the weight contours, the constraint boundaries, and the fact that a straight line drawn between the two designs passes through infeasible design space indicate that the two designs are distinct, converged optima.

Figure 9b shows the individual constraints that form the envelope constraint boundary that is shown in Figure 9a. For simplicity, weight contours are not shown in Figure 9b. Optimum design 1 is bounded by rising weight, by the flutter constraint, and by the stress constraints from elements 5 and 46. Optimum design 2 is bounded by rising weight, by the flutter constraint, and by stress constraints from elements 6,27 , and 46 .

\section{Probabilistic optimization}

The reliabilities of the two optimum deterministic designs were calculated using the probabilistic allowables given in Table 2. For designs 1 and 2, the reliabilities are 0.8967 and 0.8990 , respectively.

Probabilistic designs were obtained using, as starting points, both deterministic optima. The two starting points produced two different probabilistic designs; the probabilistic designs are similar to the original deterministic designs. The probabilistic designs are given the same designation as the deterministic design starting points - that is, optimum probabilistic design 1 was obtained using, as a starting point, optimum deterministic design 1. Optimum probabilistic design 1 
Table 4. Quantities associated with optimum probabilistic design 1. System reliability requirement is 0.9990

\begin{tabular}{|l|c|}
\hline Quantity & \multicolumn{1}{|c|}{ Value } \\
\hline Nominal Weight, lb & 31.7659 \\
\hline Mean Weight, lb & 31.8982 \\
\hline $\mathrm{P}_{\mathrm{f}}$ (stress) & $\mathbf{0 . 0 0 1 0 0 2}$ \\
\hline $\mathrm{P}_{\mathrm{f}}$ (flutter) & $\mathbf{0 . 0 0 0 0 0 6}$ \\
\hline $\mathrm{P}_{\mathrm{f}}$ (system) & $\mathbf{0 . 0 0 1 0 0 8}$ \\
\hline $\begin{array}{c}\text { Design variables } \\
\mathrm{t}_{1}\end{array}$ \\
\hline $\mathrm{t}_{2}$ & $\mathbf{0 . 1 5 5 9 0 8}$ \\
\hline $\mathrm{t}_{3}$ & $\mathbf{0 . 1 5 1 9 9 4}$ \\
\hline $\mathrm{t}_{4}$ & $\mathbf{0 . 1 5 1 3 9 9}$ \\
\hline $\mathrm{t}_{5}$ & $\mathbf{0 . 1 5 0 0 0 0}$ \\
\hline $\mathrm{t}_{6}$ & $\mathbf{0 . 5 7 9 3 6 6}$ \\
\hline $\mathrm{t}_{7}$ & $\mathbf{0 . 1 5 0 2 4 5}$ \\
\hline $\mathrm{t}_{8}$ & $\mathbf{0 . 1 5 0 0 0 0}$ \\
\hline $\mathrm{t}_{9}$ & $\mathbf{0 . 5 9 4 5 0 0}$ \\
\hline
\end{tabular}

was obtained for one reliability-0.9990. Optimum probabilistic design 2 was obtained for four reliabilities $-0.9000,0.9900,0.9990$, and 0.9999 . For each design, the weight, the probability of various modes of failure, and the values of the design variables are given in Tables 4 and 5 for designs 1 and 2 , respectively. Note that, in the tables, the quantity $P_{f}$ (stress) is the probability of failure by exceeding the allowable stress in any element. (Optimum probabilistic designs could have been obtained for several values of the reliability for either probabilistic design 1 or probabilistic design 2 . Design 2 was selected to demonstrate the technology in this paper.)

In Tables 4 and 5 and elsewhere in this paper, the quantity "Nominal Weight" is the weight calculated using the deterministic thickness design variables $t_{i}$ rather than the random thicknesses $t_{i}$ (random). The quantity "Mean Weight" is the mean value of the weight calculated using the random thicknesses $t_{i}$ (random). Random thicknesses are always used in calculating the reliability.

The nominal and mean weights of design 2 (Table 5) are presented as a function of probability of system failure $\left(\mathrm{P}_{\mathrm{f}}\right)$ and reliability in Figure 10. Five thousand Monte Carlo simulations were used to determine the mean weight. Note that the horizontal scale is logarithmic with respect to $P_{f}$. Below the values of $P_{f}$ are the corresponding values of the reliability. Plotted in this manner, the nominal and mean weights are almost linear with respect to both $\mathrm{P}_{\mathrm{f}}$ and to the reliability. The curves show the difference between the two weights-about $0.12 \mathrm{lb}$ or about 0.38 percent. The curves also show that large increases in reliability can be obtained with relatively small increases in weight.

Table 5. Quantities associated with optimum pmbabilistic design 2

for four system reliability requirements

\begin{tabular}{|l|r|r|r|r|}
\hline \multirow{2}{*}{ Quantity } & \multicolumn{4}{|c|}{ System Reliability Requirement } \\
\cline { 2 - 5 } & $\mathbf{0 . 9 0 0 0}$ & $\mathbf{0 . 9 9 0 0}$ & $\mathbf{0 . 9 9 9 0}$ & $\mathbf{0 . 9 9 9 9}$ \\
\hline Nominal Weight, lb & 30.7185 & 31.2389 & 31.7918 & 32.3702 \\
\hline Mean Weight, lb & 30.8500 & 31.3715 & 31.9277 & 32.5073 \\
\hline $\mathrm{P}_{\mathrm{f}}$ (stress) & $\mathbf{0 . 0 5 1 3 9 0}$ & $\mathbf{0 . 0 0 9 7 7 4}$ & $\mathbf{0 . 0 0 0 0 1 2}$ & $\mathbf{0 . 0 0 0 1 0 0}$ \\
\hline $\mathrm{P}_{\mathrm{f}}$ (flutter) & $\mathbf{0 . 0 5 1 9 1 0}$ & $\mathbf{0 . 0 0 0 2 3 0}$ & $\mathbf{0 . 0 0 0 9 8 6}$ & $\mathbf{0 . 0 0 0 0 0 0}$ \\
\hline $\mathrm{P}_{\mathrm{f}}$ (system) & $\mathbf{0 . 1 0 0 0 0 1}$ & $\mathbf{0 . 0 1 0 0 0 3}$ & $\mathbf{0 . 0 0 0 9 9 8}$ & $\mathbf{0 . 0 0 0 1 0 0}$ \\
\hline Design variables $_{\mathrm{t}_{\mathbf{1}}}$ & $\mathbf{0 . 1 5 0 0 0 0}$ & $\mathbf{0 . 1 5 0 0 5 4}$ & $\mathbf{0 . 1 5 0 5 9 2}$ & $\mathbf{0 . 1 5 3 0 0 4}$ \\
\hline $\mathrm{t}_{\mathbf{2}}$ & $\mathbf{0 . 4 2 9 7 3 5}$ & $\mathbf{0 . 4 4 0 1 6 4}$ & $\mathbf{0 . 4 5 7 5 5 6}$ & $\mathbf{0 . 4 6 7 1 9 4}$ \\
\hline $\mathrm{t}_{3}$ & $\mathbf{0 . 5 1 5 6 4 1}$ & $\mathbf{0 . 5 2 5 4 6 6}$ & $\mathbf{0 . 5 3 6 4 5 2}$ & $\mathbf{0 . 5 4 7 3 7 3}$ \\
\hline $\mathrm{t}_{\mathbf{4}}$ & $\mathbf{0 . 5 7 2 6 8 9}$ & $\mathbf{0 . 5 6 8 4 9 8}$ & $\mathbf{0 . 5 5 5 3 8 2}$ & $\mathbf{0 . 5 6 3 1 9 8}$ \\
\hline $\mathrm{t}_{5}$ & $\mathbf{0 . 2 0 7 6 8 2}$ & $\mathbf{0 . 2 1 7 4 2 4}$ & $\mathbf{0 . 2 2 6 7 2 6}$ & $\mathbf{0 . 2 3 2 4 7 6}$ \\
\hline $\mathrm{t}_{\mathbf{6}}$ & $\mathbf{0 . 1 5 0 1 3 9}$ & $\mathbf{0 . 1 5 0 1 2 3}$ & $\mathbf{0 . 1 5 4 5 8 3}$ & $\mathbf{0 . 1 5 7 3 5 0}$ \\
\hline $\mathrm{t}_{7}$ & $\mathbf{0 . 1 6 7 2 6 7}$ & $\mathbf{0 . 1 5 7 1 6} 9$ & $\mathbf{0 . 1 5 0 2 4 3}$ & $\mathbf{0 . 1 5 0 0 0 0}$ \\
\hline $\mathrm{t}_{8}$ & $\mathbf{0 . 1 5 0 0 9 1}$ & $\mathbf{0 . 1 5 0 0 0 0}$ & $\mathbf{0 . 1 5 0 4 8 7}$ & $\mathbf{0 . 1 5 0 0 7 2}$ \\
\hline $\mathrm{t}_{9}$ & $\mathbf{0 . 1 5 2 2 0 5}$ & $\mathbf{0 . 1 5 0 9 0 5}$ & $\mathbf{0 . 1 5 1 1 1 0}$ & $\mathbf{0 . 1 5 0 6 7 5}$ \\
\hline
\end{tabular}




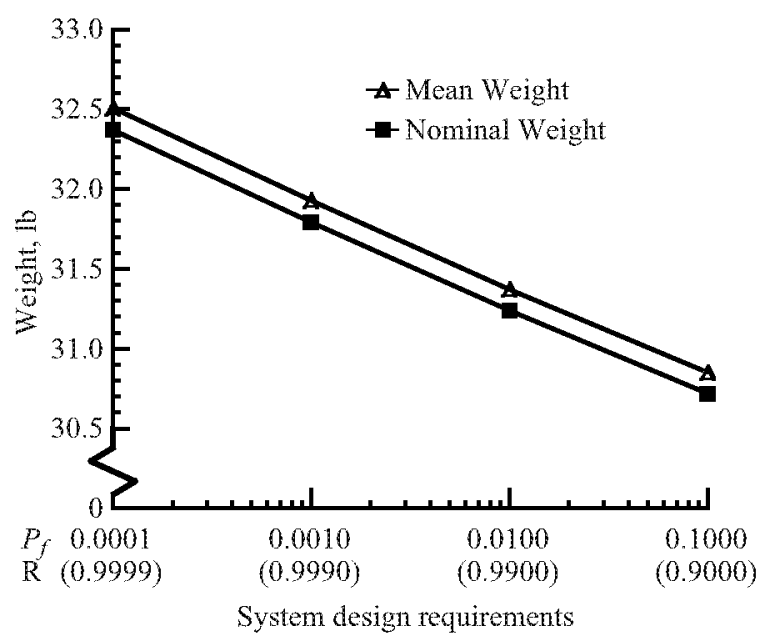

Figure 10. Nominal and mean weights of optimum probabilistic design 2 as a function of system requirement on probability of failure $\left(\mathrm{P}_{\mathrm{f}}\right)$ and reliability $(\mathbf{R})$. $\mathbf{R}=\mathbf{1}-\mathbf{P}_{\mathrm{f}}$.

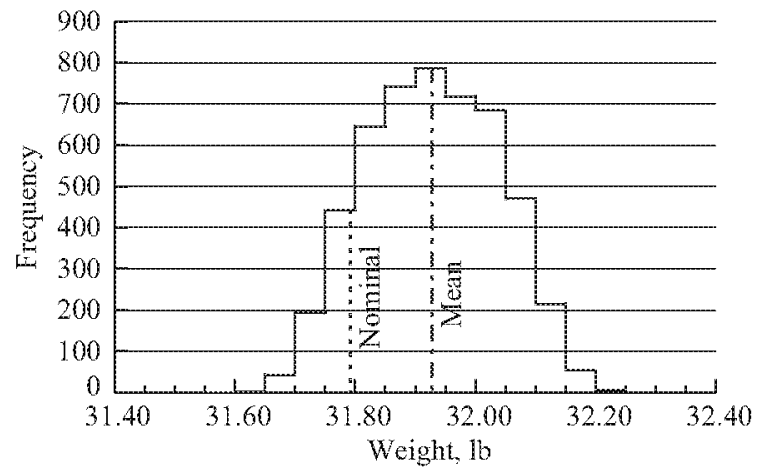

Figure 11. Distribution of weights produced by $\mathrm{t}_{\mathrm{i}}($ random $)$ for design 2 , reliability $=\mathbf{0 . 9 9 9 0}$.

In Tables 4 and 5, six significant digits are presented because that precision is needed to obtain a minimum weight design for a reliability of $\mathbf{0 . 9 9 9 9}$. To be consistent, six significant digits are presented for all values of design reliability.

The range of weights produced by $t_{i}$ (random) is indicated in Figure 11. These data are for design 2, system reliability $=\mathbf{0} .9990$. Five thousand Monte Carlo simulations were used to determine the distribution. The nominal weight and the mean weight are also shown. The range of possible weights is greater than the difference between the nominal and mean weights.
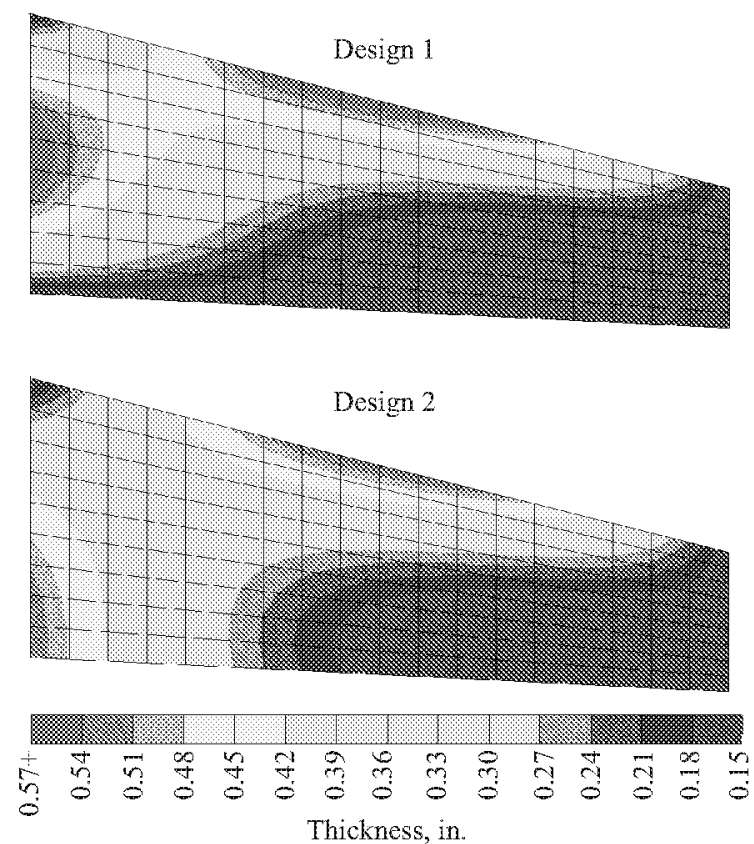

Figure 12. Thickness distribution for optimum probabilistic designs 1 and 2. System reliability requirement is 0.9990 .

Contour plots that show the thickness distributions of probabilistic designs 1 and 2 for a reliability of 0.9990 are presented in Figure 12. These thickness distributions were calculated using the deterministic design variables $t_{i}$ rather than the random thicknesses $t_{i}$ (random). If the same terminology that was used above to describe the weight is used here, the thickness distributions shown in Figure 12 could be referred to as Nominal Thicknesses rather than Mean Thicknesses. These thickness distributions are similar to those in Figure 7 for the deterministic designs.

Figure 13 provides detailed information about the stresses in probabilistic designs 1 and 2 that were designed for a reliability of 0.9990 . This figure indicates the probability that the von Mises stress exceeds the allowable stress at the centroid of each finite element. Whereas Tables 4 and 5 give the probability of exceeding the allowable stress in any element, Figure 13 gives the probability of failure for each element. The results show that there are only a few elements where the stress has a relatively high likelihood of exceeding the allowable stress. These elements are the same as those that provide the active constraints for the deterministic designs. 

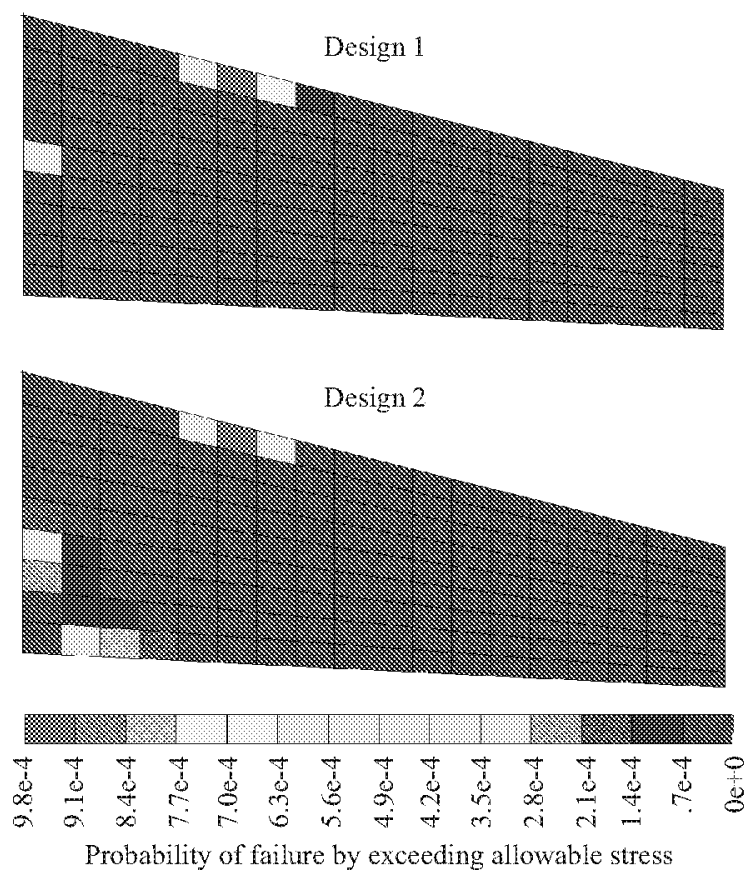

Figure 13. Probability that von Mises stress exceeds the allowable stress at the centroid of each finite element. Designs are optimum probabilistic designs 1 and 2 for system reliability requirement of $\mathbf{0 . 9 9 9 0}$.
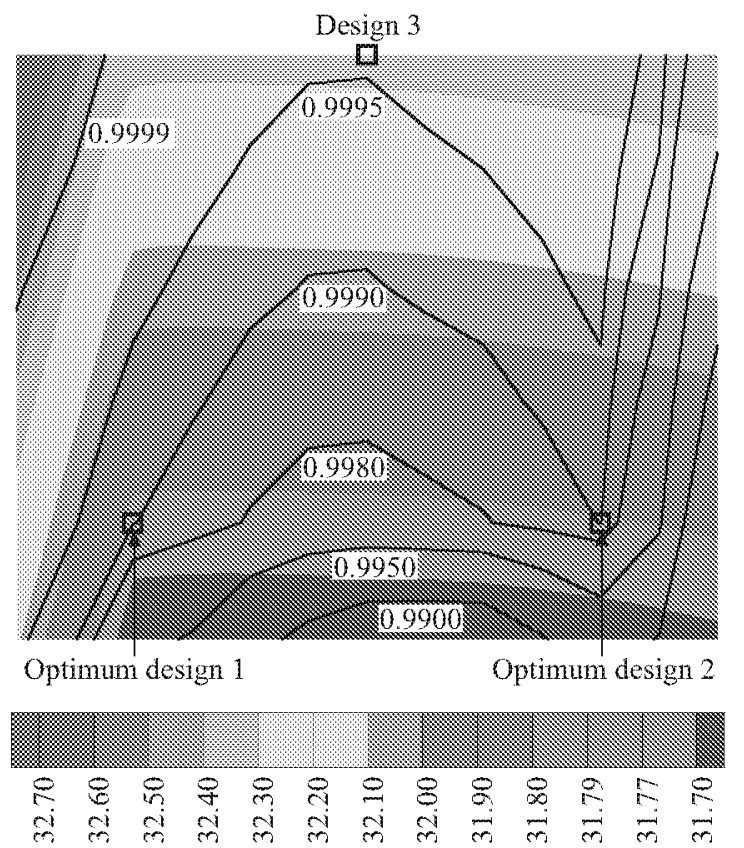

Weight, $1 \mathrm{~b}$

Figure 14. Plane through design space with optimum probabilistic designs 1 and 2 , weight contours, and curves of constant system reliability.
Finally, a chart similar to Figure 9 is shown in Figure 14 to help explain the differences between probabilistic designs 1 and 2 for a reliability of 0.9990 . The third design in Figure 14 was obtained the same way it was obtained for Figure 9. The difference between Figure 9 and Figure 14 is that in Figure 14 the constraint curves are system reliability. Although the design space in Figure 14 is not exactly the same as in Figure 9, it is close enough to see how various failure modes affect the reliability. For example, the sharp changes in the reliability in the right portion of Figure 14 are caused by the multiple stress failure modes shown near design 2 in Figure 9 b.

\section{Concluding Remarks}

An approach for carrying out reliability-based design of a metal, plate-like wing that can serve as the center portion and primary stiffness of a wind tunnel model is presented. The plate is designed to meet strength and flutter requirements defined in terms of risk/reliability. Although this study does not address all the issues associated with designing this type of wind tunnel model, this study can serve as the first step in developing a capability to account for uncertainties in the design of such a wind tunnel model. The design approach can also be used for preliminary design of wing structures for aircraft with thin wings.

The design problem that was studied can be defined as follows. Determine the thickness distribution such that wing weight is a minimum and the probability of failune is less than a specified value. Failune is assumed to occur if either the flutter speed is less than a specified allowable or the stress caused by a pressure loading is greater than a specified allowable. There are four uncertainties: wing thickness, calculated flutter speed, allowable stress, and magnitude of a uniform pressure load. Data for uncertainties in the thickness distribution of the metal wing were obtained from a NASA machine shop that has made such models.

The key steps of the design approach are as follows. The reliability-based design optimization started with a design obtained using conventional deterministic design optimization with margins on the allowables. The reliability was calculated using Monte Carlo simulation (MCS) with one million trials. MCS interacted with response surfaces that provided values of stresses and flutter speed. During the reliability-based design optimization, the response surfaces and move limits were coordinated to ensure accuracy of the response surfaces. 
The studies produced two reliability-based designs that met the design requirements and had nearly the same weight. A novel graphical approach is used to show how the two designs are distinct, converged optima.

The studies showed the relationship between reliability and weight and showed that, for the design problem considered, large increases in reliability could be obtained with relatively small increases in weight.

\section{Appendix A. Mathematical Expression that Represents Wing Thickness Distribution}

The thickness varies over the wing as a biquadratic polynomial in $\mathrm{x}$ and $\mathrm{y}$ as follows

$$
\begin{aligned}
t= & a_{1}+a_{2} x+a_{3} x y+a_{4} y+a_{5} x^{2}+a_{6} x^{2} y \\
& +a_{7} x y^{2}+a_{8} y^{2}+a_{9} x^{2} y^{2}
\end{aligned}
$$

The unknown coefficients $a_{i}(i=1,9)$ in Equation (A1) can be determined from thickness values at nine locations in the wing. For the present study, the nine locations are shown in Figure 2 and in Figure A1(a). Using Lagrange finite element shape functions and the thickness at these nine locations on the wing, the thickness distribution $t(\xi, \eta)$ can be described in a curvilinear coordinate system (Fig. $\mathrm{A1}(\mathrm{b}))$ as

$$
\begin{aligned}
t(\xi, \eta)= & \left(c_{1}+c_{2} \eta+c_{3} \eta^{2}\right)+\left(c_{4}+c_{5} \eta+c_{6} \eta^{2}\right) \xi \\
& +\left(c_{7}+c_{8} \eta+c_{9} \eta^{2}\right) \xi^{2}
\end{aligned}
$$

where

$$
\begin{gathered}
c_{1}=t_{5} \\
c_{2}=\frac{1}{2}\left(t_{8}-t_{2}\right) \\
c_{3}=\frac{1}{2}\left(t_{2}+t_{8}\right)-t_{5} \\
c_{4}=\frac{1}{2}\left(t_{6}-t_{4}\right) \\
c_{5}=\frac{1}{4}\left(t_{1}+t_{9}-t_{3}-t_{7}\right) \\
=\frac{1}{2}\left(t_{4}-t_{6}\right)+\frac{1}{4}\left(t_{3}+t_{9}-t_{1}-\right. \\
c_{7}=\frac{1}{2}\left(t_{4}+t_{6}\right)-t_{5} \\
c_{9}=t_{5}-\frac{1}{2}\left(t_{2}+t_{4}+t_{6}+t_{8}\right) \\
=\frac{1}{2}\left(t_{2}-t_{8}\right)+\frac{1}{4}\left(t_{7}+t_{9}-t_{1}-\right.
\end{gathered}
$$$$
c_{6}=\frac{1}{2}\left(t_{4}-t_{6}\right)+\frac{1}{4}\left(t_{3}+t_{9}-t_{1}-t_{7}\right)
$$$$
c_{8}=\frac{1}{2}\left(t_{2}-t_{8}\right)+\frac{1}{4}\left(t_{7}+t_{9}-t_{1}-t_{3}\right)
$$

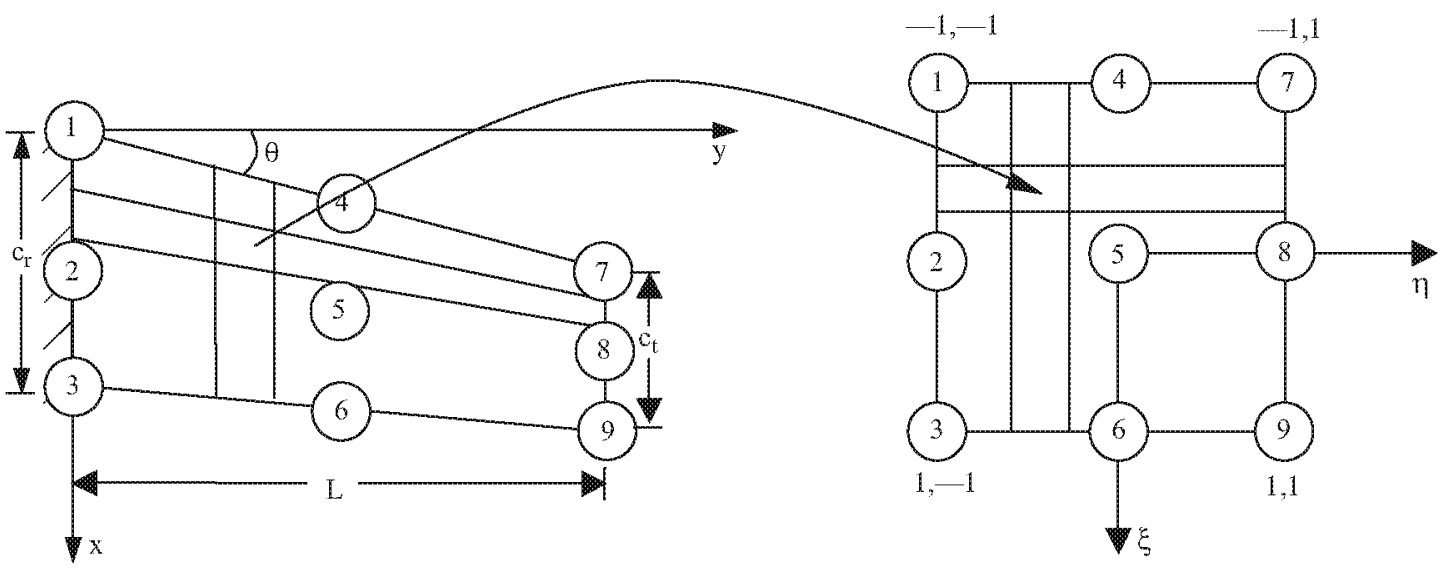

(a) Wing planform in Cartesian ( $x, y)$ coordinate system. (b) Location of nodes in curvilinear $(\xi, \eta)$ system.

Figure A1. Wing planform and Lagrange isoparametric transformation. 
The equation relating $(\mathrm{x}, \mathrm{y})$ and $(\xi, \eta)$ can be written as

$$
\begin{gathered}
\eta=\frac{2 y}{L}-1 \\
\xi=\frac{2 L(x-y \tan \theta)}{c_{r} L-\left(c_{r}-c_{t}\right) y}-1
\end{gathered}
$$

The quantities $\mathrm{L}, \theta, \mathrm{c}_{\mathrm{r}}$, and $\mathrm{c}_{\mathrm{t}}$ in Equations (A12) and (A13) are shown in Figure A1(a).

When response surfaces were created for stress and flutter, the wing was modeled using 162, eight-node quadrilateral finite elements. The finite element analysis code MSC/NASTRAN requires the thickness at each of the four comer nodes of each quadrilateral finite element as input. The thicknesses of the comer nodes were obtained using Equation (A4) for a given set of thickness $\left(t_{1}\right.$ to $\left.t_{9}\right)$. However, if a calculated thickness is less than the minimum thickness 0.15 in., the thickness at that node is set equal to 0.15 in.

\section{Appendix B. Origin of Randomness in Thickness-Machine Shop Measurements}

Data describing the uncertainties associated with machining a wind tunnel model were obtained from quality control measurements of a wind tunnel model that had been machined and measured at NASA Langley Research Center. These measurements were taken at 14,805 points and were compared with the plans provided by the customer, the test engineer. The plans called for a tolerance of $\mathbf{\pm 0 . 0 0 2 5}$ in

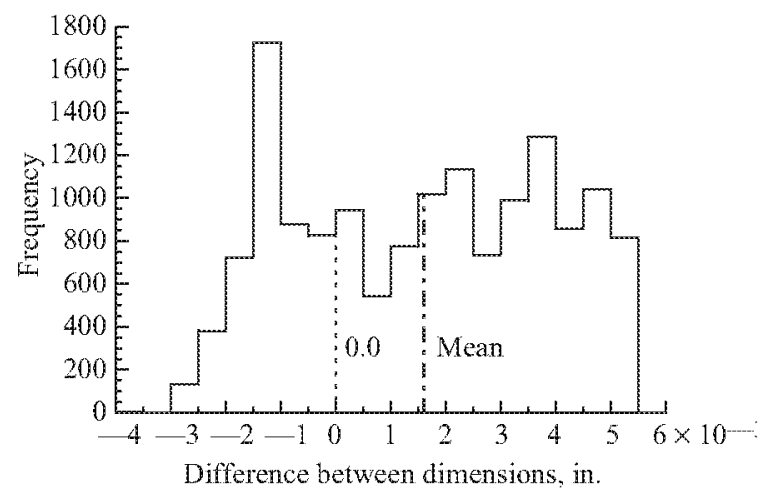

Figure B1. Distribution of difference between measured dimensions and a specified dimension for a wind tunnel model. Difference is given by measured dimension minus specified dimension.
Figure B1 presents a histogram of the differences between a measured dimension and the specified dimension. Positive numbers indicate an excess of material. The results show that the number of points with excess material, and the magnitude of the excesses, exceeds the number and magnitude of the undercuts. This distribution reflects the approach used to produce the model. After the model was machined and canefully measured, the model and measurements were shown to the customer. The customer could accept the job, or could request some rework. Since it was much easier to rework the job by removing material than by adding material, it was much more likely that the machinist would leave excess material than undercut the job. Therefore, the distribution shown in Figure B1 is not symmetric about a difference of zero.

Regarding the undercut data at the left in Figure $\mathrm{B} 1$, since the customer specified a tolerance of $\pm \mathbf{0 . 0 0 2 5}$ in., only a small number of occurrences fell outside that tolerance. The data in Figure B1 was approximated by a uniform distribution between $\mathbf{- 0 . 0 0 2 5}$ and $+\mathbf{0 . 0 0 5 5}$, which is shown in Figure 5 .

\section{Appendix C. Response Surface Used in Calculations}

In this paper, Monte Carlo simulation (MCS) is used to calculate the reliability. Unfortunately, MCS requires a large number of function evaluations to obtain the required accuracy. Because the stress and flutter analyses used in this paper are computationally expensive, it would not be practical to perform exact analyses for every simulation. Instead, an altemate method was used to calculate the stresses and flutter speed. That method was based on response surface approximations

In the response surface approach, approximate polynomial functions are fit to the response over the design space. MCS interacts with these relatively simple functions rather than with the more complex exact analyses. In the present work, one quadratic polynomial is used to represent the stress response in each of the 162 finite elements, and one quadratic polynomial is used to represent the flutter speed, for a total of 163 response surfaces. A quadratic polynomial with 9 design variables $(v)$ requires $k=(v+1)(v+2) / 2=55$ coefficients.

In this study, the 55 coefficients of the polynomial were estimated by using a face-centered central composite design approach (Ref. 11). In this approach, design points are established at the center of the design 
space, at the comers of the design space (the intersection between each of the design variable limits), and at the face-centers of the design space (points determined by setting one variable to its upper or lower limit with all other variables set to the average of their upper and lower limits). The number of design points, $p$, required by this pocedure is $\mathrm{p}=2^{\mathrm{v}}+2 \mathrm{v}+1=531$ points. The method of least squares is used to solve for the 55 coefficients by minimizing the emor between the 531 design points and the response polynomial.

\section{References}

1. Rudisill, Carl S., and Bhatia, Kumar G., "Optimization of Complex Structures to Satisfy Flutter Requirements," AIAA Journal, Vol. 9, pp. 1487-1491, 1971.

2. Stroud, W. Jefferson, Dexter, Cornelia B., and Stein, Manuel, "Automated Preliminary Design of Simplified Wing Structures to Satisfy Strength and Flutter Requirements," NASA TN D-6534, 1971.

3. McCullers, L. A., and Lynch, R. W., "Composite Wing Design for Aeroelastic Requirements," Proceedings of the Conference on Fibrous Composites in Flight Vehicle Design, AFFDL-TR-72-130, 1972.

4. Haftka, Raphael T., "Automated Procedure for Design of Wing Structures to Satisfy Strength and Flutter Requirements," NASA TN D-7264, 1973.

5. Caffrey, John P., and Lee, John N., M SC/ NASTRAN Linear Static Analysis, User's Guide,
V68, The MacNeal-Schwendler Corporation, Los Angeles, CA, 1996.

6. Rodden, William P., and Johnson, Erwin H., MSC/NASTRAN Aeroelastic Analysis, User's Guide, V68, The MacNeal-Schwendler Corporation, Los Angeles, CA, 1994.

7. Anonymous, DOT, Design Optimization Tools, Users Manual, Version 5.0, Vanderplaats Research \& Development, Inc., Colorado Springs, CO 80906.

8. Ang, Alfredo H.-S., and Tang, Wilson H., Probability Concepts in Engineering Planning and Design. Vol. I, Basic Principles, John Wiley and Sons, N.Y., 1975

9. Ang, Alfredo H.-S., and Tang, Wilson H., Probability Concepts in Engineering Planning and Design. Vol. II, Decision, Risk, and Reliability, John Wiley and Sons, N.Y., 1984.

10. Knill, Duane L., Giunta, Anthony A., Baker, Chuck A., Grossman, Bemard, Mason, William H., Haftka, Raphael T., and Watson, Layne T., "Response Surface Models Combining Linear and Euler Aerodynamics for Supersonic Transport Design.” AIAA Journal of Aircraft, Vol. 36, No. 1, January-February 1999.

11. Myers, Raymond $H$. and Montgomery, Douglas C., Response Surface Methodology, Process and Product Optimization Using Designed Experiments, John Wiley and Sons, N.Y., 1995. 